PROCEEDINGS OF THE

AMERICAN MATHEMATICAL SOCIETY

Volume 135, Number 5, May 2007, Pages 1431-1440

S 0002-9939(06)08614-X

Article electronically published on November 13, 2006

\title{
SPECTRAL LOCALIZATION IN THE HIERARCHICAL ANDERSON MODEL
}

\author{
EVGENIJ KRITCHEVSKI
}

(Communicated by Mikhail Shubin)

\begin{abstract}
We prove that a large class of hierarchical Anderson models with spectral dimension $\mathrm{d} \leq 2$ has only pure point spectrum.
\end{abstract}

\section{INTRODUCTION}

This paper is devoted to the study of the spectral properties of the hierarchical Anderson model and is motivated by the work of Molchanov [M2]. Before stating our results we recall the definition of the model and its basic properties. For additional information about the hierarchical structures and the hierarchical Anderson model we refer the reader to $[\mathrm{D}, \mathrm{BS}, \mathrm{BO}, \mathrm{M} 1, \mathrm{M} 2$.

Let $X$ be an infinite countable set. Throughout the paper $\delta_{x}$ will denote the Kronecker delta function at $x \in X$. A partition $\mathcal{P}$ of $X$ is a collection of its disjoint subsets whose union is equal to $X$. Let $\mathbf{n}=\left(n_{r}\right)_{r>0}$ be a sequence of positive integers and $\mathbf{P}=\left(\mathcal{P}_{r}\right)_{r \geq 0}$ a sequence of partitions of $X$. The elements of $\mathcal{P}_{r}$ are called "clusters" of rank $r$. We say that $(X, \mathbf{P}, \mathbf{n})$ is a hierarchical structure if the following hold:

(1) $n_{0}=1$ and every $Q \in \mathcal{P}_{0}$ has exactly one element.

(2) For $r \geq 1$, every $Q \in \mathcal{P}_{r}$ is a disjoint union of $n_{r}$ clusters in $\mathcal{P}_{r-1}$.

(3) Given $x, y \in X$, there is a cluster $Q$ of some rank containing both $x$ and $y$. Let us state some immediate consequences of this definition. Every cluster of rank $r \geq 0$ has size $N_{r}:=\prod_{s=0}^{r} n_{s}$. Given $x \in X$ and $r \geq 0$, there is a unique cluster of rank $r$ containing $x$. We denote this cluster by $Q_{r}(x)$. The map

$$
d(x, y):=\min \left\{r: y \in Q_{r}(x)\right\}
$$

is a metric on $X$ and $Q_{r}(x)=\{y: d(x, y) \leq r\}$. Note that $Q_{r}(x)=Q_{r}(y)$ whenever $d(x, y) \leq r$. Given an integer $n \geq 2$, a hierarchical structure is called homogeneous of degree $n$ if $n_{r}=n$ for all $r \geq 1$.

The free Laplacian on the hierarchical structure $(X, \mathbf{P}, \mathbf{n})$ is defined as follows.

For each $r \geq 0$, let $E_{r}: l^{2}(X) \rightarrow l^{2}(X)$ be the averaging operator

$$
\left(E_{r} \psi\right)(x):=\frac{1}{N_{r}} \sum_{d(x, y) \leq r} \psi(y) .
$$

Received by the editors December 8, 2005.

2000 Mathematics Subject Classification. Primary 47B80, 47A55, 93A13.

This work was supported in part by an FQRNT grant.

(C)2006 American Mathematical Society Reverts to public domain 28 years from publication 
Let $\mathbf{p}=\left(p_{r}\right)_{r \geq 1}$ be a sequence of positive numbers such that $\sum_{r=1}^{\infty} p_{r}=1$. In the sequel we set $p_{0}:=0$ and

$$
\lambda_{r}:=\sum_{s=0}^{r} p_{s}, \quad r=0,1, \cdots, \infty .
$$

The hierarchical Laplacian $\Delta$ on $l^{2}(X)$ is defined by

$$
\Delta:=\sum_{r=0}^{\infty} p_{r} E_{r}
$$

Clearly, $\Delta$ is a bounded self-adjoint operator and $0 \leq \Delta \leq 1$.

A hierarchical model is a hierarchical structure $(X, \mathbf{P}, \mathbf{n})$ together with the hierarchical Laplacian $\Delta$. The spectral properties of $\Delta$ only depend on $\mathbf{n}$ and $\mathbf{p}$ and are summarized in:

Theorem 1.1. (1) The spectrum of $\Delta$ is equal to $\left\{\lambda_{r}: r=0, \cdots, \infty\right\}$. Each $\lambda_{r}$, $r<\infty$, is an eigenvalue of $\Delta$ of infinite multiplicity. The point $\lambda_{\infty}=1$ is not an eigenvalue.

(2) $E_{r}-E_{r+1}$ is the orthogonal projection onto the eigenspace of $\lambda_{r}$ and

$$
\Delta=\sum_{r=0}^{\infty} \lambda_{r}\left(E_{r}-E_{r+1}\right) .
$$

(3) For every $x \in X$, the spectral measure for $\delta_{x}$ and $\Delta$ is given by

$$
\mu=\sum_{r=0}^{\infty}\left(\frac{1}{N_{r}}-\frac{1}{N_{r+1}}\right) \delta\left(\lambda_{r}\right),
$$

where $\delta\left(\lambda_{r}\right)$ stands for the Dirac unit mass at $\lambda_{r}$. Note that $\mu$ does not depend on $x$.

The spectral measure $\mu$ can be naturally interpreted as the integrated density of states of the operator $\Delta$. Let $x_{0} \in X$ be given and consider the increasing sequence of clusters $Q_{r}\left(x_{0}\right), r \geq 0$. Let $P_{r}$ be the orthogonal projection onto the $N_{r}$-dimensional subspace

$$
l^{2}\left(Q_{r}\left(x_{0}\right)\right):=\left\{\psi \in l^{2}(X): \psi(x)=0 \text { for } x \notin Q_{r}\left(x_{0}\right)\right\} .
$$

Let $e_{1}^{(r)} \leq e_{2}^{(r)} \leq \cdots \leq e_{N_{r}}^{(r)}$ be the eigenvalues of the restricted Laplacian $P_{r} \Delta P_{r}$ acting on $l^{2}\left(Q_{r}\left(x_{0}\right)\right)$ and

$$
\nu_{r}:=\frac{1}{N_{r}} \sum_{s=1}^{r} \delta\left(e_{s}^{(r)}\right)
$$

the corresponding counting measure.

Proposition 1.2. The weak-* limit $\lim _{r \rightarrow \infty} \nu_{r}$ exists and is equal to $\mu$.

If

$$
\lim _{t \downarrow 0} \frac{\log \mu([1-t, 1])}{\log t}=\mathrm{d} / 2,
$$

then the number $\mathrm{d}$ is called the spectral dimension of $\Delta$. This definition is motivated by the analogy with the edge asymptotics of the density of states of the standard discrete Laplacian on $\mathbb{Z}^{\mathrm{d}}$, for which the spectral and spatial dimensions coincide.

The relation $\sum_{y \in X}\left\langle\delta_{x} \mid \Delta \delta_{y}\right\rangle=1$ yields that $\Delta$ generates a random walk on $X$. We recall that the random walk on $\mathbb{Z}^{\mathrm{d}}$ generated by the standard discrete 
Laplacian is recurrent if $\mathrm{d}=1,2$ and transient if $\mathrm{d}>2$. The corresponding result for the hierarchical Laplacian is:

Proposition 1.3. Consider a homogeneous hierarchical structure of degree $n \geq 2$. Suppose that there exist constants $C_{1}>0, C_{2}>0$ and $\rho>1$ such that

$$
C_{1} \rho^{-r} \leq p_{r} \leq C_{2} \rho^{-r}
$$

for $r$ big enough. Then:

(1) The spectral dimension of this model is

$$
\mathrm{d}(n, \rho)=2 \frac{\log n}{\log \rho} .
$$

Hence $0<\mathrm{d}(n, \rho) \leq 2$ iff $n \leq \rho$.

(2) The random walk generated by $\Delta$ is recurrent if $0<\mathrm{d}(n, \rho) \leq 2$ and transient if $\mathrm{d}(n, \rho)>2$.

We now define the hierarchical Anderson model associated to $(X, \mathbf{P}, \mathbf{n})$ and the hierarchical Laplacian $\Delta$. Consider the probability space $(\Omega, \mathcal{F}, \mathbb{P})$ where $\Omega:=\mathbb{R}^{X}$, $\mathcal{F}$ is the usual Borel $\sigma$-algebra in $\Omega$, and $\mathbb{P}$ is a given probability measure on $(\Omega, \mathcal{F})$. For $\omega \in \Omega$, we set

$$
V_{\omega}:=\sum_{x \in X} \omega(x)\left\langle\delta_{x} \mid \cdot\right\rangle \delta_{x} .
$$

$V_{\omega}$ is a self-adjoint (possibly unbounded) multiplication operator on $l^{2}(X)$. Let

$$
H_{\omega}:=\Delta+V_{\omega}, \quad \omega \in \Omega .
$$

The family of self-adjoint operators $\left\{H_{\omega}\right\}_{\omega \in \Omega}$ indexed by the events of the probability space $(\Omega, \mathcal{F}, \mathbb{P})$ is called the hierarchical Anderson model.

Concerning the probability measure $\mathbb{P}$, we will need only one technical assumption having to do with the notion of conditional density. Throughout the paper, $m$ will denote the Lebesgue measure on $\mathbb{R}$. For any $x \in X, \Omega$ can be decomposed along the $x$ th coordinate as $\Omega=\mathbb{R} \times \widetilde{\Omega}, \widetilde{\Omega}=\mathbb{R}^{X \backslash\{x\}}$. Let $\widetilde{\mathbb{P}}_{x}$ be the corresponding marginal of $\mathbb{P}$ defined by $\widetilde{\mathbb{P}}_{x}(\widetilde{B}):=\mathbb{P}(\mathbb{R} \times \widetilde{B})$, where $\widetilde{B} \subset \widetilde{\Omega}$ is a Borel set. Then for $\widetilde{\mathbb{P}}_{x}$-a.e. $\widetilde{\omega} \in \widetilde{\Omega}$, there is a probability measure $\mathbb{P}_{x}^{\tilde{\omega}}$ on $\mathbb{R}$ s.t. the conditional Fubini theorem holds: for all $f \in L^{1}(\Omega, P)$ we have

$$
\int_{\Omega} f(\omega) d \mathbb{P}(\omega)=\int_{\widetilde{\Omega}}\left(\int_{\mathbb{R}} f(\xi, \widetilde{\omega}) d \mathbb{P}_{x}^{\tilde{\omega}}(\xi)\right) d \widetilde{\mathbb{P}}_{x}(\widetilde{\omega}) .
$$

If for $\widetilde{\mathbb{P}}_{x}$-a.e. $\widetilde{\omega} \in \widetilde{\Omega}, \mathbb{P}_{x}^{\tilde{\omega}}$ is absolutely continuous (a.c.) with respect to $m$, then we say that $\mathbb{P}$ has a conditional density along the $x$ th coordinate. $\mathbb{P}$ is called conditionally a.c. if for every $x \in X, \mathbb{P}$ has a conditional density along the $x$ th coordinate. An important special case of a conditionally a.c. probability measure is the product measure $\mathbb{P}=\bigotimes_{x \in X} \mathbb{P}_{x}$, where each $\mathbb{P}_{x}$ is a probability measure on $\mathbb{R}$ a.c. with respect to $m$.

We denote by $\sigma_{\mathrm{ac}}\left(H_{\omega}\right)$ the absolutely continuous part of the spectrum of $H_{\omega}$ and by $\sigma_{\text {cont }}\left(H_{\omega}\right)$ the continuous part. Our main result is:

Theorem 1.4. Assume that there exists a sequence $u_{r}>0$ such that $\sum_{r=1}^{\infty} u_{r}^{-1}<$ $\infty$ and

$$
\sum_{r=1}^{\infty} p_{r} N_{r-1} u_{r-1} u_{r}<\infty
$$


Then:

(1) For all $\omega \in \Omega, \sigma_{\mathrm{ac}}\left(H_{\omega}\right)=\emptyset$.

(2) If $\mathbb{P}$ is conditionally a.c., then $\sigma_{\text {cont }}\left(H_{\omega}\right)=\emptyset$ for $\mathbb{P}$-a.e. $\omega$.

Remark 1. Theorem 1.4 and Proposition 1.3 allow us to construct hierarchical models with spectral dimension $\mathrm{d} \leq 2$ that exhibit Anderson localization at arbitrary disorder. If $(X, \mathbf{P}, \mathbf{n})$ is a homogeneous hierarchical structure of degree $n \geq 2$ and $p_{r}=C \rho^{-r}$ with $\rho>n$, then the hypothesis (1.1) is fulfilled for $u_{r}=r^{1+\varepsilon}$. Given $0<\mathrm{d}<2$, one can adjust $\rho>n$ to make $\mathrm{d}(n, \rho)=\mathrm{d}$. If $p_{r}=C r^{-3-\varepsilon} n^{-r}$, then the model has spectral dimension $\mathrm{d}=2$ and (1.1) is verified for $u_{r}=r^{1+\varepsilon / 3}$. One can also construct trivial models with $\mathrm{d}=0$ by taking $p_{r}$ to decrease faster than $\rho^{-r}$ for any $\rho$. We emphasize that homogeneity of the hierarchical structure is not required for Theorem 1.4

Remark 2. In [M2, Molchanov has proven that if the random variables $\omega(x)$ are i.i.d. with a Cauchy distribution, then Theorem 1.4 holds under the condition

$$
\sum_{r=1}^{\infty} p_{r} u_{r}<\infty
$$

In particular, in this case the theorem holds for $\Delta$ of any spectral dimension. Molchanov's argument is based on subtle properties of Cauchy random variables and cannot be directly extended to any other probability measure. In contrast, our proof of localization in spectral dimension $\mathrm{d} \leq 2$ is based on general arguments and is the first step in extending Molchanov's result to a more general class of probability measures.

Remark 3. The fractional moments method of Aizenman and Molchanov [AM] allows us to prove localization for $\Delta+\sigma V_{\omega}$ for large disorder $\sigma$ or for large energies. One needs an extra decoupling hyphothesis on the random variables $\omega(x)$ and the condition on $\Delta$ that

$$
B:=\sup _{x} \sum_{y \in X}\left|\left\langle\delta_{x} \mid \Delta \delta_{y}\right\rangle\right|^{s}<\infty
$$

for some $0<s<1$. Simple estimates show that

$$
\sum_{r=1}^{\infty} p_{r} N_{r}^{1-s} \leq B \leq \sum_{r=1}^{\infty} p_{r}^{s} N_{r}^{1-s} .
$$

The requirement (1.2) on the decay of $p_{r}$ is comparable to the hypothesis (1.1), while Theorem 1.4 is valid at arbitrary disorder or energy.

Remark 4. Part (2) of Theorem 1.4 does not hold for all $\omega$. Our method of proof combined with the general results of [DMS, G] yields that $H_{\omega}$ will have singular continuous spectrum for some $\omega$ 's.

\section{THE FREE LAPLACIAN}

In this section, we prove Theorem 1.1, Proposition 1.2 and Proposition 1.3

Proof of Theorem 1.1. For $r \geq 0$, let $\mathcal{H}_{r}=\operatorname{Ran}\left(E_{r}\right)$. $\mathcal{H}_{r}$ is the closed subspace of $l^{2}(X)$ consisting of functions that are constant on each cluster of rank $r$. Note that

$$
l^{2}(X)=\mathcal{H}_{0} \supset \mathcal{H}_{1} \supset \mathcal{H}_{2} \supset \mathcal{H}_{3} \supset \ldots
$$


and that $\bigcap \mathcal{H}_{r}=\{0\}$ since a nonzero function constant on every cluster would have infinite $l^{2}$ norm. These observations yield that

$$
l^{2}(X)=\bigoplus_{r=0}^{\infty} L_{r}
$$

where $L_{r}$ is the orthogonal complement of $\mathcal{H}_{r+1}$ in $\mathcal{H}_{r}$. Note that $L_{r}$ is the infinitedimensional subspace of functions $\psi$ s.t. $E_{s} \psi=\psi$ for $0 \leq s \leq r$ and $E_{s} \psi=0$ for $s>r$. Hence for every $\psi \in L_{r}, \Delta \psi=\lambda_{r} \psi$, and this proves parts (1) and (2).

The spectral measure $\mu_{x, \Delta}$ for $\delta_{x}$ and $\Delta$ is the unique Borel probability measure on $\mathbb{R}$ s.t.

$$
\left\langle\delta_{x} \mid f(\Delta) \delta_{x}\right\rangle=\int_{\mathbb{R}} f(\xi) d \mu_{x, \Delta}(\xi),
$$

for every bounded Borel function $f: \mathbb{R} \rightarrow \mathbb{C}$. To compute $\mu_{x, \Delta}$, we decompose $\delta_{x}$ according to (2.1):

$$
\delta_{x}=\sum_{r=0}^{\infty}\left(E_{r}-E_{r+1}\right) \delta_{x}=\sum_{r=0}^{\infty}\left(\frac{1}{N_{r}} \mathbf{1}_{Q_{r}(x)}-\frac{1}{N_{r+1}} \mathbf{1}_{Q_{r+1}(x)}\right),
$$

where $\mathbf{1}_{Q_{r}(x)}:=\sum_{y \in Q_{r}(x)} \delta_{y}$. Hence

$$
f(\Delta) \delta_{x}=\sum_{r=0}^{\infty} f\left(\lambda_{r}\right)\left(\frac{1}{N_{r}} \mathbf{1}_{Q_{r}(x)}-\frac{1}{N_{r+1}} \mathbf{1}_{Q_{r+1}(x)}\right)
$$

and

$$
\left\langle\delta_{x} \mid f(\Delta) \delta_{x}\right\rangle=\sum_{r=0}^{\infty} f\left(\lambda_{r}\right)\left\|\frac{1}{N_{r}} \mathbf{1}_{Q_{r}(x)}-\frac{1}{N_{r+1}} \mathbf{1}_{Q_{r+1}(x)}\right\|^{2} .
$$

Since $\left\|\frac{1}{N_{r}} \mathbf{1}_{Q_{r}(x)}-\frac{1}{N_{r+1}} \mathbf{1}_{Q_{r+1}(x)}\right\|^{2}=1 / N_{r}-1 / N_{r+1}$, (3) follows.

The analysis of the density of states of $\Delta$ is facilitated if one introduces the cut-off Laplacians

$$
\Delta_{r}:=\sum_{s=0}^{r} p_{s} E_{s}, \quad r \geq 0 .
$$

It is technically easier to work with $\Delta_{r}$ than with $P_{r} \Delta P_{r}$. Note that $l^{2}\left(Q_{r}\left(x_{0}\right)\right)$ is an invariant subspace for $\Delta_{r}$. One can exactly compute the eigenvalues and eigenvectors of the restricted operator $P_{r} \Delta_{r}$ acting on $l^{2}\left(Q_{r}\left(x_{0}\right)\right)$. If $0 \leq s \leq r$, then every $\psi \in L_{s} \cap l^{2}\left(Q_{r}\left(x_{0}\right)\right)$ is an eigenvector of $P_{r} \Delta_{r}$ with eigenvalue $\lambda_{r}$. The subspace $L_{s} \cap l^{2}\left(Q_{r}\left(x_{0}\right)\right)$ has dimension $D_{s}^{(r)}:=N_{r}\left(1 / N_{s}-1 / N_{s+1}\right)$ for $0 \leq s \leq r-1$, and the subspace $L_{r} \cap l^{2}\left(Q_{r}\left(x_{0}\right)\right)$ has dimension $D_{r}^{(r)}:=1$. Since $\sum_{s=0}^{r} D_{s}^{(r)}=N_{r}$, the spectrum of $P_{r} \Delta_{r}$ is equal to $\left\{\lambda_{s}: s=0, \cdots, r\right\}$ and each eigenvalue $\lambda_{s}$ has multiplicity $D_{s}^{(r)}$.

Proof of Proposition 1.3. Let $\nu^{*}$ be a weak-* limit point of the sequence $\nu_{r}$. Let $\nu_{r_{k}}$ be a subsequence converging to $\nu^{*}$. We claim that

$$
\nu^{*}\left(\left\{\lambda_{s}\right\}\right)=\mu\left(\left\{\lambda_{s}\right\}\right),
$$

for all $s \geq 0$. Indeed, let $\delta:=\min _{j \neq s}\left|\lambda_{s}-\lambda_{j}\right| / 2$ and $0<\varepsilon<\delta / 3$. Since $\left\|P_{r} \Delta P_{r}-P_{r} \Delta_{r}\right\| \leq \sum_{j=r+1}^{\infty} p_{j}$, we have that $\left\|P_{r} \Delta P_{r}-P_{r} \Delta_{r}\right\| \leq \varepsilon$ for all $r$ big enough. For such $r$, the spectrum of $P_{r} \Delta P_{r}$ is contained in $\bigcup_{j=0}^{r}\left[\lambda_{j}-\varepsilon, \lambda_{j}+\varepsilon\right]$. Let $R$ be the spectral projection of $P_{r} \Delta P_{r}$ on $\left[\lambda_{s}-\varepsilon, \lambda_{s}+\varepsilon\right]$ and $T$ the spectral 
projection of $P_{r} \Delta_{r}$ on the same interval. Let $\gamma$ be the circle $\left\{z \in \mathbb{C}:\left|z-\lambda_{s}\right|=\delta\right\}$, oriented counterclockwise. Then

$$
\begin{aligned}
R-T & =\frac{1}{2 \pi i} \oint_{\gamma}\left(z-P_{r} \Delta P_{r}\right)^{-1} d z-\frac{1}{2 \pi i} \oint_{\gamma}\left(z-P_{r} \Delta_{r}\right)^{-1} d z \\
& =\frac{1}{2 \pi i} \oint_{\gamma}\left(z-P_{r} \Delta P_{r}\right)^{-1}\left(P_{r} \Delta P_{r}-P_{r} \Delta_{r}\right)\left(z-P_{r} \Delta_{r}\right)^{-1} d z,
\end{aligned}
$$

and thus

$$
\|R-T\| \leq \delta(2 \delta / 3)^{-1} \varepsilon(2 \delta / 3)^{-1} \leq 3 / 4<1 .
$$

It follows that $\operatorname{Ran}(R)$ and $\operatorname{Ran}(T)$ have the same dimension and that

$$
\#\left\{s: e_{s}^{(r)} \in\left[\lambda_{s}-\varepsilon, \lambda_{s}+\varepsilon\right]\right\}=D_{s}^{(r)} .
$$

Then for all $k$ big enough

$$
\nu_{r_{k}}\left(\left[\lambda_{s}-\varepsilon, \lambda_{s}+\varepsilon\right]\right)=D_{s}^{(r)} / N_{r}=1 / N_{s}-1 / N_{s+1} .
$$

Letting $k \rightarrow \infty$, we get $\nu^{*}\left(\left[\lambda_{s}-\varepsilon, \lambda_{s}+\varepsilon\right]\right)=1 / N_{s}-1 / N_{s+1}$, and (2.2) follows by taking $\varepsilon \downarrow 0$. Since $\sum_{s=0}^{\infty}\left(1 / N_{s}-1 / N_{s+1}\right)=1$ and $\nu^{*}$ is a probability measure, we must have that $\nu^{*}=\mu$. Therefore $\mu$ is the unique weak-* limit point of the sequence $\nu_{r}$ and $\lim _{r \rightarrow \infty} \nu_{r}=\mu$.

Proof of Proposition [1.3. Note that $\mu([1-t, 1])$ is a piecewise constant function of $t$ with jump discontinuities at the points $1-\lambda_{r}$. Since

$$
C_{1}(\rho-1)^{-1} \rho^{-r} \leq 1-\lambda_{r}=\sum_{s=r+1}^{\infty} p_{s} \leq C_{2}(\rho-1)^{-1} \rho^{-r},
$$

and $\mu\left(\left[1-\lambda_{r}, 1\right]\right)=1 / N_{r}=n^{-r}$, we have that

$$
\lim _{t \downarrow 0} \frac{\log \mu([1-t, 1])}{\log t}=\frac{\log n}{\log \rho}
$$

which proves (1).

The random walk on $X$ starting at $x$ is transient if $R:=\sum_{k=0}^{\infty}\left\langle\delta_{x} \mid \Delta^{k} \delta_{x}\right\rangle<\infty$ and recurrent if $R=\infty$. Part (3) of Theorem 1.1 allows us to compute $R$ explicitly:

$$
R=\left\langle\delta_{x} \mid(1-\Delta)^{-1} \delta_{x}\right\rangle=\int \frac{d \mu(\xi)}{1-\xi}=\sum_{r=0}^{\infty} \frac{N_{r}^{-1}-N_{r+1}^{-1}}{1-\lambda_{r}} .
$$

The bounds

$$
C_{2}^{-1}(\rho-1)(1-1 / n) \sum_{r=0}^{\infty}(\rho / n)^{r} \leq R \leq C_{1}^{-1}(\rho-1)(1-1 / n) \sum_{r=0}^{\infty}(\rho / n)^{r}
$$

show that $R<\infty$ for $\rho<n$ and $R=\infty$ for $\rho \geq n$, and part (2) follows.

\section{Proof of the localization theorem}

This section is devoted to the proof of Theorem 1.4 and is organized as follows. We first derive a hierarchical approximation formula for the resolvent $\left(H_{\omega}-z\right)^{-1}$. Then we use the formula to obtain a bound on the resolvent matrix elements. This bound combined with the Simon-Wolff localization criterion yields the statement. Set

$$
H_{\omega, r}:=V_{\omega}+\sum_{s=0}^{r} p_{s} E_{s}, \quad r \geq 0 .
$$


Fix $\omega \in \Omega$. For any $Q_{r} \in \mathcal{P}_{r}$, the subspace $l^{2}\left(Q_{r}\right)$ is invariant for $H_{\omega, r}$. Let $\sigma\left(\omega, Q_{r}\right)$ be the set of the eigenvalues of the restricted operator $H_{\omega, r} \uparrow l^{2}\left(Q_{r}\right)$ and $\sigma_{\omega}:=\bigcup \sigma\left(\omega, Q_{r}\right)$ where the union is over all clusters of all ranks. Clearly, $\sigma_{\omega}$ is a countable subset of $\mathbb{R}$. For $z \in \mathbb{C} \backslash \sigma_{\omega}, r \geq 0$, and $x, y \in X$, we set

$$
G_{\omega, r}(x, y ; z):=\left\langle\delta_{x} \mid\left(H_{\omega, r}-z\right)^{-1} \delta_{y}\right\rangle .
$$

For $z \in \mathbb{C} \backslash \sigma_{\omega}, r \geq 0$ and $t \in X$, let $g_{\omega, r}(t ; z)$ be the average of $G_{\omega, r}(\cdot, t ; z)$ over the cluster $Q_{r}(t)$, i.e.

$$
g_{\omega, r}(t ; z):=\frac{1}{N_{r}} \sum_{d\left(t^{\prime}, t\right) \leq r} G_{\omega, r}\left(t^{\prime}, t ; z\right) .
$$

Since the joint spectral measure for $\delta_{t}, \delta_{t^{\prime}}$ and $H_{\omega, r}$ is real, $G_{\omega, r}\left(t^{\prime}, t ; z\right)=$ $G_{\omega, r}\left(t, t^{\prime} ; z\right)$ and

$$
g_{\omega, r}(t ; z)=\frac{1}{N_{r}} \sum_{d\left(t^{\prime}, t\right) \leq r} G_{\omega, r}\left(t, t^{\prime} ; z\right)=\frac{1}{N_{r}}\left\langle\delta_{t} \mid\left(H_{\omega, r}-z\right)^{-1} \mathbf{1}_{Q_{r}(t)}\right\rangle .
$$

Proposition 3.1. Let $\omega \in \Omega, x, y \in X, z \in \mathbb{C} \backslash \sigma_{\omega}$ and $r \geq 0$ be given. Then

$$
G_{\omega, r}(x, y ; z)=G_{\omega, 0}(x, y ; z)-\sum_{s=d(x, y)}^{r} p_{s} N_{s-1} g_{\omega, s-1}(x ; z) g_{\omega, s}(y ; z) .
$$

Proof. The formula holds for $r=0$ since $p_{0}=0$. For $s \geq 1$, the resolvent identity yields

$$
\left(H_{\omega, s}-z\right)^{-1} \delta_{y}-\left(H_{\omega, s-1}-z\right)^{-1} \delta_{y}=-\left(H_{\omega, s-1}-z\right)^{-1} p_{s} E_{s}\left(H_{\omega, s}-z\right)^{-1} \delta_{y} .
$$

Observe that $E_{s}\left(H_{\omega, s}-z\right)^{-1} \delta_{y}=g_{\omega, s}(y ; z) \mathbf{1}_{Q_{s}(y)}$. Taking $\left\langle\delta_{x} \mid \cdot\right\rangle$ in the above equation yields

$$
G_{\omega, s}(x, y ; z)-G_{\omega, s-1}(x, y ; z)=-p_{s} g_{\omega, s}(y ; z)\left\langle\delta_{x} \mid\left(H_{\omega, s-1}-z\right)^{-1} \mathbf{1}_{Q_{s}(y)}\right\rangle .
$$

Note that by (3.1),

$$
\left\langle\delta_{x} \mid\left(H_{\omega, s-1}-z\right)^{-1} \mathbf{1}_{Q_{s}(y)}\right\rangle= \begin{cases}N_{s-1} g_{\omega, s-1}(x ; z), & \text { if } d(x, y) \leq s, \\ 0, & \text { if } d(x, y)>s .\end{cases}
$$

The formula (3.2) follows after adding (3.3) for $s=1,2, \cdots, r$.

The key step in our proof is:

Theorem 3.2. Suppose that $p_{r}$ and $N_{r}$ satisfy (1.1). Let $\omega \in \Omega$ and $x \in X$ be fixed. Then for $m$-a.e. $e \in \mathbb{R} \backslash \sigma_{\omega}$,

$$
\sup _{r \geq 0} \sum_{y \in X}\left|G_{\omega, r}(x, y ; e)\right|^{2}<\infty .
$$

Proof. We shall use the following general result, proven in [M2]:

Let $A$ be a hermitian $N \times N$ matrix and $v \in \mathbb{C}^{N}$. Then for all $M>0$,

$$
m\left(\left\{e:\left\|(A-e)^{-1} v\right\|_{2}^{2} \geq M\right\}\right) \leq 4 \sqrt{\frac{N}{M}}\|v\|_{2},
$$

where $\|\cdot\|_{2}$ stands for the $l^{2}$ norm on $\mathbb{C}^{N}$. 
Since $l^{2}\left(Q_{r}(x)\right)$ is an $N_{r}$-dimensional invariant subspace for $H_{\omega, r}$ and since $\left\|\mathbf{1}_{Q_{r}(x)}\right\|_{2}=\sqrt{N_{r}}$, we have from (3.5) that for $M_{r}>0$,

$$
m\left(\left\{e \in \mathbb{R} \backslash \sigma_{\omega}:\left\|\left(H_{\omega, r}-e\right)^{-1} \mathbf{1}_{Q_{r}(x)}\right\|_{2}^{2} \geq M_{r}\right\}\right) \leq \frac{4 N_{r}}{\sqrt{M_{r}}} .
$$

Let $M_{r}>0$ be a sequence satisfying $\sum_{r=1}^{\infty} N_{r} M_{r}^{-1 / 2}<\infty$. By the Borel-Cantelli lemma, for $m$-a.e. $e \in \mathbb{R} \backslash \sigma_{\omega}$, there exists a finite constant $C_{e}$ such that

$$
\left\|\left(H_{\omega, r}-e\right)^{-1} \mathbf{1}_{Q_{r}(x)}\right\|_{2}^{2}<C_{e} M_{r},
$$

for all $r \geq 0$. From now on, such an $e \in \mathbb{R} \backslash \sigma_{\omega}$ is fixed. Using the representation formula (3.2), we get the estimate

$$
\begin{aligned}
\left(\sum_{y \in X}\left|G_{\omega, r}(x, y ; e)\right|^{2}\right)^{1 / 2} & \leq\left|G_{\omega, 0}(x, x ; e)\right| \\
& +\sum_{s=1}^{r} p_{s} N_{s-1}\left|g_{\omega, s-1}(x ; e)\right|\left(\sum_{d(x, y) \leq s}\left|g_{\omega, s}(y ; e)\right|^{2}\right)^{1 / 2}
\end{aligned}
$$

Observe that

$$
\begin{aligned}
\left(\sum_{d(x, y) \leq s}\left|g_{\omega, s}(y ; e)\right|^{2}\right)^{1 / 2} & =\left(\sum_{d(x, y) \leq s}\left|\frac{1}{N_{s}}\left\langle\delta_{y} \mid\left(H_{\omega, s}-e\right)^{-1} \mathbf{1}_{Q_{s}(y)}\right\rangle\right|^{2}\right)^{1 / 2} \\
& =\frac{1}{N_{s}}\left(\sum_{d(x, y) \leq s}\left|\left\langle\delta_{y} \mid\left(H_{\omega, s}-e\right)^{-1} \mathbf{1}_{Q_{s}(x)}\right\rangle\right|^{2}\right)^{1 / 2} \\
& =\frac{1}{N_{s}}\left\|\left(H_{\omega, s}-e\right)^{-1} \mathbf{1}_{Q_{s}(x)}\right\|_{2} .
\end{aligned}
$$

Inequality (3.6) gives the bound

$$
\left(\sum_{d(x, y) \leq s}\left|g_{\omega, s}(y ; e)\right|^{2}\right)^{1 / 2} \leq C_{e}^{1 / 2} \frac{\sqrt{M_{s}}}{N_{s}} .
$$

Moreover

$$
N_{s-1}\left|g_{\omega, s-1}(x ; e)\right|=\left|\left\langle\delta_{x} \mid\left(H_{\omega, s-1}-e\right)^{-1} \mathbf{1}_{Q_{s-1}(x)}\right\rangle\right| \leq C_{e}^{1 / 2} \sqrt{M_{s-1}} .
$$

Combining (3.7) with (3.9) and (3.8) yields the estimate

$$
\left(\sum_{y \in X}\left|G_{\omega, r}(x, y ; e)\right|^{2}\right)^{1 / 2} \leq\left|G_{\omega, 0}(x, x ; e)\right|+C_{e} \sum_{s=1}^{r} p_{s} \frac{\sqrt{M_{s}} \sqrt{M_{s-1}}}{N_{s}} .
$$

By hypothesis (1.1), the sequence $M_{r}=\left(u_{r} N_{r}\right)^{2}$ satisfies

$$
\sum_{r=1}^{\infty} N_{r} M_{r}^{-1 / 2}=\sum_{r=1}^{\infty} u_{r}^{-1}<\infty .
$$


Since

$$
\sum_{r=1}^{\infty} p_{r} \frac{\sqrt{M_{r}} \sqrt{M_{r-1}}}{N_{r}}=\sum_{r=1}^{\infty} p_{r} N_{r-1} u_{r-1} u_{r}<\infty
$$

the result follows.

Let us recall the Simon-Wolff localization criterion. For $x \in X$ and $\omega \in \Omega$, denote by $\mu_{x}^{\omega}$ the spectral measure for $\Delta+V_{\omega}$ and $\delta_{x}$, by $\mu_{x, \text { cont }}^{\omega}$ the continuous part of $\mu_{x}^{\omega}$ and by $\mu_{x, \text { ac }}^{\omega}$ the a.c. part. Define the function $G_{\omega, x}: \mathbb{R} \rightarrow[0,+\infty]$ by

$$
G_{\omega, x}(e):=\int_{\mathbb{R}} \frac{d \mu_{x}^{\omega}(\lambda)}{(e-\lambda)^{2}}=\lim _{\epsilon \downarrow 0}\left\|\left(\Delta+V_{\omega}-e-i \epsilon\right)^{-1} \delta_{x}\right\|^{2} .
$$

By the Theorem of de la Vallé Poussin,

$$
d \mu_{x, \mathrm{ac}}^{\omega}(e)=\pi^{-1}\left(\lim _{\epsilon \downarrow 0} \varepsilon\left\|\left(\Delta+V_{\omega}-e-i \epsilon\right)^{-1} \delta_{x}\right\|^{2}\right) d e .
$$

Hence, if for a fixed $\omega \in \Omega$ we have that $G_{\omega, x}(e)<\infty$ for $m$-a.e. $e \in \mathbb{R}$, then $\mu_{x, \text { ac }}^{\omega}=0$.

The Simon-Wolff localization criterion is summarized in:

Theorem 3.3. Assume that $\mathbb{P}$ has a conditional density along the $x$ th coordinate. Let $B \subset \mathbb{R}$ be a Borel set such that $G_{\omega, x}(e)<\infty$ for $\mathbb{P} \otimes m$-a.e. $(\omega, e) \in \Omega \times B$. Then $\mu_{x, \text { cont }}^{\omega}(B)=0$ for $\mathbb{P}$-a.e. $\omega \in \Omega$.

Theorem 3.3 is a well-known consequence of the rank-1 Simon-Wolff theorem [SW] and the conditional Fubini theorem.

Proof of Theorem 1.4. Fix $\omega \in \Omega$ and fix $e \in \mathbb{R} \backslash \sigma_{\omega}$ for which the bound (3.4) holds. By monotone convergence

$$
\int_{\mathbb{R}} \frac{d \mu_{x}^{\omega}(\lambda)}{(e-\lambda)^{2}}=\lim _{\varepsilon \downarrow 0} \int_{\mathbb{R}} \frac{d \mu_{x}^{\omega}(\lambda)}{(e-\lambda)^{2}+\varepsilon^{2}}=\sup _{\varepsilon>0} \int_{\mathbb{R}} \frac{d \mu_{x}^{\omega}(\lambda)}{(e-\lambda)^{2}+\varepsilon^{2}} .
$$

Since for any $z \in \mathbb{C} \backslash \mathbb{R}$,

$$
\lim _{r \rightarrow \infty}\left\|\left(H_{\omega, r}-z\right)^{-1}-\left(H_{\omega}-z\right)^{-1}\right\|=0,
$$

we have that the weak-* $\operatorname{limit}_{\lim _{r \rightarrow \infty}} \mu_{x, r}^{\omega}$ equals $\mu_{x}^{\omega}$, where $\mu_{x, r}^{\omega}$ is the spectral measure for $H_{\omega, r}$ and $\delta_{x}$. Therefore

$$
\begin{aligned}
\int_{\mathbb{R}} \frac{d \mu_{x}^{\omega}(\lambda)}{(e-\lambda)^{2}}=\sup _{\varepsilon>0} \lim _{r \rightarrow \infty} \int_{\mathbb{R}} \frac{d \mu_{x}^{\omega, r}(\lambda)}{(e-\lambda)^{2}+\varepsilon^{2}} & \leq \sup _{\varepsilon>0, r \geq 1} \int_{\mathbb{R}} \frac{d \mu_{x}^{\omega, r}(\lambda)}{(e-\lambda)^{2}+\varepsilon^{2}} \\
& =\sup _{r \geq 1} \int_{\mathbb{R}} \frac{d \mu_{x}^{\omega, r}(\lambda)}{(e-\lambda)^{2}} \\
& =\sup _{r \geq 1}\left\|\left(H_{\omega, r}-e\right)^{-1} \delta_{x}\right\|^{2} \\
& =\sup _{r \geq 1} \sum_{y \in X}\left|G_{\omega, r}(x, y ; e)\right|^{2}<\infty .
\end{aligned}
$$

In the final equality we used the fact that $\left\{\delta_{y}: y \in X\right\}$ is an orthonormal basis for $l^{2}(X)$. Since $m\left(\sigma_{\omega}\right)=0$ and since the bound (3.4) holds for $m$-a.e. $e \in \mathbb{R} \backslash \sigma_{\omega}$, we have that for every fixed $\omega \in \Omega, G_{\omega, x}(e)<\infty$ for $m$-a.e. $e \in \mathbb{R}$. This proves part (1). Part (2) follows from the fact that $G_{\omega, x}(e)<\infty$ for $\mathbb{P} \otimes m$-a.e. $(\omega, e) \in \Omega \times \mathbb{R}$ and the Simon-Wolff criterion. 


\section{ACKNOWLEDGEMENTS}

We are grateful to Professor Vojkan Jaksic, who suggested this research project, and from whom the author learned about random Schrödinger operators. We would like to thank Professor Stanislav Molchanov for very helpful discussions and encouragements during the author's visit at the University of Charlotte, NC. We also benefited from discussions with the following people: Kingwood Chen, Serguei Denissov, Marco Merkli, Juan-Manuel Perez-Abarca and Nicola Squartini. A very special thanks goes to Kingwood Chen for hospitality during the author's visit at UNCC.

\section{REFERENCES}

[AM] Aizenman, M., Molchanov, S.: Localization at large disorder and at extreme energies: an elementary derivation. Commun. Math. Phys. 157 (1993), no. 2, 245-278. MR.1244867 (95a:82052)

[Bo] Bovier, A.: The density of states in the Anderson model at weak disorder: a renormalization group analysis of the hierarchical model. J. Statist. Phys. 59 (1990), no. 3-4, 745-779. MR1063180 (91m:82063)

[BS] Bleher, P. M., Sinai, Ya. G.: Investigation of the Critical Point in Models of the Type of Dyson's Hierarchical Models. Commun. Math. Phys. 33, (1973).

[DMS] del Rio, R., Makarov, N., Simon, B.: Operators with singular continuous spectrum: II. Rank one operators. Commun. Math. Phys. 165 (1994), 59. MR:1298942 (97a:47002)

[D] Dyson, F.J.: Existence of a phase-transition in a one dimensional Ising Ferromagnet. Comm. Math. Phys. 12, 91 (1969). MR0436850 (55:9786)

[G] Gordon, A.: Pure point spectrum under 1-parameter perturbations and instability of Anderson localization. Commun. Math. Phys. 164 (1994), 489. MR1291242 (95k:47019)

[M1] Molchanov, S.: Lectures on random media. Lectures on probability theory (Saint-Flour, 1992), 242-411, Lecture Notes in Math., 1581, Springer, Berlin, 1994. MR1307415 (95m:60165)

[M2] Molchanov, S.: Hierarchical random matrices and operators. Application to Anderson model. Multidimensional statistical analysis and theory of random matrices (Bowling Green, OH, 1996), 179-194, VSP, Utrecht, 1996. MR.1463464 (99b:82054)

[SW] Simon, B., Wolff, T.: Singular continuous spectrum under rank one perturbations and localization for random Hamiltonians. Communications in Pure and Applied Mathematics 49 (1986), 75. MR0820340 (87k:47032)

Department of Mathematics and Statistics, McGill University, 805 Sherbrooke Street West, Montreal, Quebec, Canada H3A 2K6

E-mail address: ekritc@math.mcgill.ca 